\section{Papillomviren als Krebserreger}

Zusammenfassung. Die vorliegende Arbeit beschreibt die historische Entwicklung und den heutigen Stand der Papillom-Virus-Forschung. In den letzten Jahren konnte vor allem durch methodische Fortschritte ein enormer Wissenszuwachs in diesem Bereich verzeichnet werden. So gilt heute die pathogenetische Rolle der Papillomviren bei der Entstehung des Zervixkarzinoms als gesichert. Die Virustypen HPV16 und HPV18 wurden 1995 von der "International Agency for Research on Cancer" als Humankanzerogene klassifiziert. Die Interaktion der viralen Onkogene E6 und E7 mit den Tumorsuppressorproteinen p53 und pR6 ist für die Tumorentstehung von Bedeutung. Es konnte gezeigt werden, daß durch Virusonkogene die Expression von Zytokinen und Wachstumsfaktoren wie Cyclin E und A, c-fos und $c$-jun moduliert wird und daß die Wirkung chemischer Onkogene durch Virusinfektionen synergistisch verstärkt werden kann. Schwerpunkt gegenwärtiger Forschungsprojekte ist die Entwicklung und klinische Anwendung geeigneter Vaccine gegen humane Papillomviren.

Papilloma Viruses as Cancer Pathogens. This review article describes the history and the present state of Humanpapilloma Virus (HPV) research. Considerable progress in this field has been achieved during recent years due to methodological advances in molecular biology. Based on experimental and clinical data it can be assumed that local HPV infection play a causal role in carcinogenesis. This applies in particular to cervical cancer. The International Agency for Research on Cancer declared HPV16 and HPV18 as carcinogenic to humans in 1995. Interactions of viral oncoproteins E6 and E7 with tumorsuppressor proteins p53 and pR6 provide a better understanding of tumor formation and tumor growth. Evidence is provided that viral oncoproteins stimulated the expression of various cytokines such as cycline E and A, c-fos and c-jun. Chemical carcinogenes and viral infections promote synergistically tumor development and growth in experimental settings. Present and future research is directed towards development of suitable vaccines and finally their clinical application.

Geburtsh. u. Frauenheilk. 58 (1998) 291-296

(c) Georg Thieme Verlag Stuttgart · New York

\section{H. zur Hausen}

Deutsches Krebsforschungszentrum, Heidelberg

\section{Einführung}

Im Jahre 1907 zeigte Ciuffo in Rom über einen Selbstversuch, daß Warzen des Menschen eine infektiöse Ursache haben. Diesem Versuch waren entsprechende Analysen mit Warzen bei Tieren vorausgegangen, die schon annähernd 10 Jahre früher gezeigt hatten, daß etwa Warzen bei Hunden und Rindern durch Infektionserreger bedingt waren. Gut 15 Jahre nach den Ciuffoschen Selbstversuchen, die insgesamt wegen der damals gering eingeschätzten klinischen Bedeutung von Warzen wenig Aufmerksamkeit fanden, beschrieben Lewandowsky und Lutz in Basel ein eigentümliches Krankheitsbild eine ausgeprägte, offensichtlich hereditär bedingte Verrucosis, die sie als Epidermodysplasia verruciformis bezeichneten, ohne dabei zu diesem Zeitpunkt von einer infektiösen Komponente auszugehen.

Anfang der dreißiger Jahre beschrieb dann Shope (1933) eine Papillomatose bei amerikanischen Wildkaninchen, die sich auch auf Hauskaninchen übertragen ließ und dort Papillome hervorrief, die nach mehreren Monaten häufig in Plattenepithelkarzinome konvertierten. Auch bei den Wildkaninchen traten solche Karzinome auf, wenn auch wesentlich seltener. Durch die systematischen Untersuchungen von Peyton Rous, der schon 1911 die Übertragbarkeit von Geflügelsarkomen dokumentiert hatte, wurde die karzinogene Wirkung dieser Viren eindrucksvoll belegt, wobei als interessanter Zusatzbefund sich ein synergistischer Effekt mit chemischen krebserzeugenden Substanzen nachweisen ließ (Rous u. Beard, 1934, 1935, Rous u. Friedewald, 1944).

Erst im Jahre 1949 wurden Papillomvirus-Partikel elektronenoptisch dargestellt (Strauss et al., 1949). Es vergingen weitere 14 Jahre, bevor deren Genom als doppelsträngiges DNA-Ringmolekül mit etwa 7800 Basenpaaren bestimmt war (Crawford u. Crawford, 1963). In den Jahren 1960 und 1961 konnten Ito und Evans zeigen, daß allein die gereinigte Nukleinsäure des Shopeschen Papillomvirus ausreichte, um bei Hauskaninchen Krebs zu erzeugen - mit anderen Worten, hier war bereits der frühe Nachweis erbracht, daß das Erbgut eines Papillomvirus als solitäres Karzinogen wirksam sein kann.

Die Schwierigkeiten in den folgenden Jahren, auch beim Menschen krebserzeugende Viren zu finden, ließen das anfängliche Interesse an diesen Befunden rasch verblassen. Wiederum vergingen fast zwei Jahrzehnte, bevor die Papillomviren breiteres Interesse fanden. Eine „Wende“ begann sich gegen Mitte der 70er Jahre abzuzeichnen. Sie hatte 
mehrere Gründe: Zum einen war es die Entwicklung der Molekularbiologie, die in diesen Jahren rasche Fortschritte machte, unter anderem die Entdeckung der sog. Restriktionsendonukleasen, die spezifische Schnitte in DNA-Molekülen durchführen können, die Entwicklung von DNA- und RNAHybridisierungstechniken und das Klonieren von DNA-Bruchstücken in bakteriellen Vektorsystemen. Der zweite Grund lag darin, daß - zum Teil basierend auf den methodischen Fortschritten - die wichtige Entdeckung gemacht wurde, daß es nicht einen Typ von humanpathogenen Papillomviren gibt, sondern eine Vielzahl unterschiedlicher Vertreter dieser Virusgruppe (Gissmann und zur Hausen, 1976, Gissmann et al., 1977, Orth et al., 1997). Waren bis dahin histologisch unterschiedliche Warzentypen als „Standortvarianten“ einer Virusinfektion angesehen worden, so kam es jetzt zu einem Paradigmenwechsel: Unterschiedliche Typen konnten auch unterschiedliche Pathogenität bedeuten, die Virusgruppe der Papillomviren gewann an Allgemeininteresse.

Gegen Mitte der 80er Jahre „explodierte“ das Interesse an Papillomviren geradezu. Grund dafür war die Isolierung neuer spezifischer humaner Papillomvirus-(HPV)Typen aus Genitalwarzen (Gissmann und zur Hausen, 1980, Gissmann et al., 1982) und vor allem die Charakterisierung neuer Typen direkt aus Gebärmutterhalskrebs-Biopsien (Dürst et al., 1983, Boshart et al., 1984). Die regelmäßige Anwesenheit von solchen HPV-Typen (insbesondere HPV 16 und 18) in Zervixkarzinomen führte $\mathrm{zu}$ einem enorm gesteigerten Interesse in diese Virusgruppe und zur Isolierung vieler zusätzlicher HPVGenotypen.

\section{Die Rolle von Papillomviren beim Krebs des Menschen}

Schon Ende der 70er Jahre erschienen erste Berichte über spezifische Papillomvirus-Typen in Karzinomen, die sich nach langer Latenzzeit bei Patienten mit Epidermodysplasia verruciformis entwickeln (Orth et al., 1978). Die Seltenheit dieses Krankheitsbildes erschwerte hier beträchtlich die Analyse kausaler Zusammenhänge.

Die ersten Berichte über eine mögliche Rolle von Papillomviren beim Zervixkarzinom erschienen schon 1974 und 1976 (zur Hausen et al., 1974, zur Hausen, 1976). Sie basierten zu dieser Phase jedoch auf Berichten über das gemeinsame Auftreten von Genitalwarzen und Gebärmutterhalskrebs oder auf der seltenen malignen Konversion von Genitalwarzen (Condylomata acuminata) und waren letztlich theoretische Überlegungen. Das Aufzeigen neuer Papillomvirus-Typen in Zervixkarzinom-Biopsien durch die gleiche Gruppe Anfang der 80er Jahre, bald gefolgt von ähnlichen Berichten über die gleichen Typen in prämalignen Veränderungen der Zervix und Vulva, in Vulva- und Peniskrebs-Biopsien, sowie im Scheidenkrebs, eröffnete eine neue Perspektive. Im Jahre 1985 ließ sich nachweisen, daß die HPV-DNA regelmäßig (wenn auch nicht immer) in die Wirtszell-DNA während der Karzinogenese eingebaut wird, wobei häufig spezifische Deletionen im Virusgenom auftreten, die vor allem. die sog. E2- und L2Region betreffen (Schwarz et al., 1985). Der darüber hinaus gehende wichtigste Befund war, daß zwei Gene des HPVGenoms, die sog. E6- und E7-Gene, in allen HPV-positiven Krebszellen aktiv sind, was eine wichtige Rolle für die Krebsentwicklung nahelegte.
Innerhalb der achtziger Jahre wurden weitere bedeutsame Eigenschaften der Papillomvirus-Typen in Zervixkarzinomen, die jetzt als „Hochrisikotypen“ ("high risk”) bezeichnet wurden, aufgezeigt: Diese Viren können ein breites Spektrum unterschiedlicher menschlicher Zellarten in der Gewebekultur „immortalisieren“, das heißt, sie zu unbegrenztem Wachstum anregen, während nicht infizierte Zellen nach mehreren Gewebekulturpassagen absterben (s. Review McDougall, 1994). Nach mehrjähriger Kultivierung solcher immortalisierter Zellen konvertieren einige dieser Kulturen spontan zu malignem Wachstum, wenn sie in immuninkompetente Tiere (Nacktmaus) heterotransplantiert werden. Dies zeigt das Potential der Hochrisiko-Typen, als solitäre Karzinogene wirksam zu werden (zur Hausen, 1994, 1996).

Wichtige neue Gesichtspunkte kamen auf, als die Gruppen um Howley und Harlow aufzeigten, daß die HPV-Onkoproteine E6 und E7 - ähnlich wie die Onkoproteine anderer DNATumorviren - spezifische zelluläre Proteine binden und funktionell verändern (Dyson et al., 1989, Werness et al., 1990). Das E6-Protein bindet das zelluläre p53, ein multifunktionelles Protein, das neben einer Rolle beim DNA-Repair, der Induktion von Apoptose, auch noch die Transkription einer Reihe von weiteren Genen reguliert. Die E6-Bindung führt zu einer funktionellen Inaktivierung und zu einer raschen Degradation dieses Proteins. E7 bindet dagegen das zelluläre $\mathrm{pRb}$ Protein, das zunächst als Tumorsuppressor beschrieben wurde und das eine wichtige Rolle in der Regulation der DNAReplikation der Zelle spielt. Die E7-Bindung verursacht hier eine verstärkte Phosphorylierung. Dadurch wird dieses Protein aus seiner Bindung mit einem Transkriptionsfaktor, E2 F, gedrängt, den es zuvor in inaktivem Zustand hält. Die Aktivierung von E2 F scheint einer der wichtigen Faktoren für die Stimulierung des Zellwachstums durch diese Virusinfektionen zu sein.

Obwohl diese Ergebnisse anfangs eine gradlinige Interpretation der wachstumsfördernden Eigenschaften dieser Viren zu erlauben schienen, haben weitere Analysen ergeben, daß für Immortalisierung und Malignisierung HPV-infizierter Zellen noch weitere eher komplexe Veränderungen ablaufen müssen, die zum Teil später erwähnt werden.

Zusätzlich zu einer großen Zahl exprimenteller Untersuchungen belegen inzwischen auch epidemiologische und seroepidemiologische Analysen die ätiologische Rolle spezifischer Papillomvirus-Typen für den Gebärmutterhalskrebs, wie auch für einen hohen Anteil anderer anogenitaler Krebsformen (Analkrebs, Vulva-, Penis- und Vaginalkrebs). Eine ConsensusKonferenz der International Agency for Research on Cancer in Lyon kam daher 1995 zu dem Schluß, "HPV16 and HPV18 are carcinogenic to humans" (IARC Monograph, Vol. 64,1995).

Neben den anogenitalen Krebsformen werden überwiegend die gleichen Papillomvirus-Typen auch in etwa $20 \%$ von oralen und laryngealen Krebsformen nachgewiesen. Auch hier läßt sich die Aktivität der E6 und E7 Gene aufzeigen, was eine ätiologische Rolle auch für diese Krebserkrankungen nahelegt. Gelegentlich finden sich diese Virus-Typen auch in vereinzelten Biopsien von Speiseröhrenkrebs. 


\section{Schwerpunkte der Papillomvirus-Forschung}

\section{Identifizierung bisher unbekannter Papillomvirus-Typen}

Innerhalb der vergangenen 15 Jahre hat sich die Papillomvirus-Gruppe in die komplexeste Gruppe humanpathogener Erreger entwickelt. Die enorme Heterogenität dieser VirusGruppe war in der Vergangenheit nicht voraussehbar. Zum gegenwärtigen Zeitpunkt sind 82 unterschiedliche Genotypen kloniert und sequenziert. Die Unterschiede zwischen einzelnen Genotypen betragen mindestens $10 \%$ in der Nukleotidfolge in der am besten konservierten Genregion, die für das Strukturprotein L1 kodiert. Darüber hinaus legen Analysen mit Hilfe der Polymerase-Kettenreaktion unter Verwendung sog. degenerierter Primerkombinationen und anschließender Sequenzanalyse des Amplifikats nahe, daß zumindest 50-70 weitere Genotypen existieren, ohne daß wir die Ursache dieser Typenvielfalt derzeit verstehen.

Ohne Frage ist es bedeutsam, diese vermutlich neuen VirusTypen weiter zu analysieren. Viele von ihnen wurden kürzlich aus Warzen von immunsupprimierten Organ-TransplantatPatienten isoliert, andere wiederum auch von nicht-immunsupprimierten Warzenträgern. Es sollte von Bedeutung sein, daß eine Reihe dieser Typen auch von Plattenepithelkarzinomen und Basalzellkrebsen der Haut isoliert wurden. Diese Tumoren weisen regelmäßig die DNA von bestimmten HPV „Haut-Typen“ auf (Shamanin et al., 1994, 1996, Berkhout et al., 1995, de Villiers et al., 1997).

Die Rolle dieser Virusinfektionen beim Hautkrebs ist zur Zeit noch unklar. Auf die Möglichkeit eines Zusammenwirkens solcher Virusinfektionen mit physikalischen Umweltfaktoren (UV-Licht) wird später kurz eingegangen.

Im Hinblick auf die kurze Zeitspanne, in der die große Zahl von neuen HPV-Typen isoliert wurde, ist die Wahrscheinlichkeit groß, daß noch nicht "das Ende der Fahnenstange“ erreicht wurde und weitere Typen auf ihre Charakterisierung warten. Damit können wir zur Zeit auch nicht die Gesamtbedeutung dieser Virusgruppe für menschliche Krebserkrankungen abschätzen. Wenn man dennoch derzeit ohne Berücksichtigung der Hautkrebserkrankungen global eine Aussage zur Bedeutung dieser Viren für Krebserkrankungen des Menschen machen will, so dürften sie an $15 \%$ der Krebserkrankungen bei Frauen und bei knapp 5\% der Krebserkrankungen bei Männern beteiligt sein. Die Wahrscheinlichkeit ist hoch, daß sich diese Zahlen in Zukunft nach oben verschieben werden.

\section{Wechselwirkung mit zellulären Proteinen}

Unser Verständnis der HPV-Pathogenität und Tumorigenität hängt in erheblichem Umfang von dem Erkennen der Wechselwirkungen viraler Onkoproteine mit Komponenten der normalen Zelle $a b$. In den vergangenen Jahren wurde es sehr klar, daß beide viralen Onkoproteine pleiotrope Effekte ausüben. Neben ihrer Wechselwirkung mit p53 und pRb wurden in den letzten Jahren weitere Bindungen an zelluläre Proteine beschrieben, was zur Komplexität der HPV-bedingten Tumorigenese beiträgt. Eine funktionelle Interferenz ließ sich für die zyklinabhängigen Kinase-Inhibitoren $\mathrm{p} 16^{\text {ink4 }}, \mathrm{p} 21^{\mathrm{CIP} 1}$ und p2 $7^{\mathrm{KIP} 1}$ aufzeigen, wobei vermutlich deren negative Regula- tion des Zellzyklus blockiert wird. Kürzlich wurde auch eine Wechselwirkung von E7- mit der M2-Pyruvat-Kinase beschrieben. Dies könnte bedeutsame Auswirkungen auf den Glukose-Stoffwechsel der HPV-infizierten Zelle haben.

Eine Änderung des Expressionsmusters zellulärer Gene wird häufig durch E6- und E7-Onkoproteine bewirkt: Dies ist teilweise bedingt durch die Freisetzung des Transkriptionsfaktors E2 F aus dem Komplex mit pRb, vermutlich sind aber auch andere Wechselwirkungen beteiligt. Unter den Genen, deren Aktivität modifiziert wird, befinden sich die Zykline $\mathrm{E}$ und A, B-myb, Telomerase, $c$-fos und $c$-jun. In allen diesen Fällen aktivieren die viralen Onkoproteine die Expression dieser zellulären Proteine. Das $\alpha$-Aktin-Gen der glatten Muskulatur wiederum wird unter den gleichen Voraussetzungen supprimiert. Es ist sehr wahrscheinlich, daß in der Zukunft eine größere Zahl zusätzlicher Gene identifiziert wird, deren Aktivität durch Funktionen der viralen Onkoproteine entweder gesteigert oder unterdrückt wird. Es ist zunehmend klar, daß diese Proteine als pleiotroper Schalter funktionieren und dabei mit einer Vielzahl zellulärer Promotoren in Wechselwirkung treten.

\section{Regulation der viralen Transkription}

Epidemiologie und einige andere Charakteristika der virusbedingten Tumorentstehung sind kaum zu verstehen, wenn wir nicht eine zelluläre Regulation der Transkription oder Funktion viraler Onkoproteine postulieren. Zu diesen Charakteristika gehören die langen Latenzzeiten zwischen Primärinfektion und Tumorauftreten, die regelmäßig mehrere Jahrzehnte betragen. Weiter sind hier zu nennen die Monoklonalität der entstehenden Tumoren, die geringe Zahl infizierter Individuen, die letztendlich diese Krebsform entwickeln und auch Wechselwirkungen zwischen Infektion und chemischen und physikalischen krebsauslösenden Faktoren (siehe unten). Sie alle deuten auf die Existenz einer wie auch immer gearteten zellulären Kontrolle der viralen Onkoproteine, die im seltenen Fall der Krebsentwicklung unterbrochen wurde.

Solche Überlegungen führen zum Konzept des zellulären Interferenzfaktors (CIF), wobei anfangs vermutet wurde, daß zumindest ein zellulärer Faktor vorliegt, der im Verlauf der Tumorprogression ausgeschaltet werden muß (zur Hausen, 1977). In den Folgejahren erwies sich, daß zumindest zwei Signalkaskaden in jeder wachstumsfähigen Zelle existieren, von denen eine die Papillomvirus-Transkription, die andere die virale Onkoprotein-Funktion supprimiert. Der CIF-Faktor mußte also konsequenterweise durch das CIF-Konzept ersetzt werden (zur Hausen, 1994).

Die funktionelle Kontrolle viraler Onkoproteine kann aus somatischen Zellhybridisierungs-Versuchen abgeleitet werden, bei denen die Fusion von zwei unterschiedlichen HPVimmortalisierten Zell-Klonen zu somatischen Hybriden führt, die sich für die Zellalterung komplementieren können, mit anderen Worten, der immortalisierte Zustand verhält sich rezessiv - trotz fortgesetzter Synthese der viralen Onkoproteine. Die transkriptionelle Kontrolle läßt sich elegant dokumentieren, wenn man immortalisierte Zellen in geeignete tierische Wirte (Nacktmaus) inokuliert. Unter diesen Bedingungen wird die in der Gewebekultur aktive Transkription der E6- und E7-Gene innerhalb von drei Tagen weitgehend unter- 
drückt (Bosch et al., 1990). Diese transkriptionelle Suppression kann auch unter Gewebekultur-Bedingungen beobachtet werden, wenn man nämlich den immortalisierten Zellen menschliche Makrophagen zusetzt (Rösl et al., 1994). Nicht ganz unerwartet übt ein Zytokin, das von Makrophagen sezerniert wird, $\mathrm{TNF} \alpha$, den gleichen Effekt aus. Eine sorgfältige Analyse des zugrunde liegenden Mechanismus legt nahe, daß TNF $\alpha$ eine Signalkaskade anschaltet, die den AP-1-Transkriptionsfaktor modifiziert. Dieser Faktor ist für die Funktion der HPV-Gene von ausschlaggebender Bedeutung. Unter Gewebekultur-Bedingungen setzt er sich aus $c$-jun/c-jun-Homodimeren oder aus $c$-jun/c-fos-Heterodimeren zusammen und aktiviert die entsprechende Genfunktion. Der Zusatz von TNF $\alpha$ führt zu einer spezifischen Phosphorylierung von c-jun und zu dessen Heterodimerisierung mit einem fos-Analogon, dem fra-1. Offensichtlich blockieren c-jun/fra-1-Heterodimere die Aktivität des HPV-Promotors.

In malignen Zellen ist die gleiche Signalkette inaktiv, die HPVGene werden im heterologen Wirtssystem aktiv transkribiert und wachsen invasiv. Offensichtlich hat hier eine Unterbrechung der Signalkette durch mutative Ereignisse in daran beteiligten zellulären Genen stattgefunden. Die maligne Progression resultiert daher als Konsequenz der wachstumsstimulierenden Eigenschaften viraler Onkoproteine und der Unterbrechung zweier vorhandener vermutlich unabhängiger zellulärer Signalketten: einer intrazellulär regulierten Kette, die mit der Funktion viraler Onkoproteine interferiert, und einer interzellulären Kette, die über andere Zellkompartimente die virale Onkogen-Transkription blockiert und die in wachstumsfähigen Zellen aktiv ist. Die Identifizierung der zellulären Gene, die diese Signalketten regulieren, ist gegenwärtig ein wichtiger Aspekt der Forschung.

\section{Wechselwirkungen mit anderen Karzinogenen}

Schon in den dreißiger Jahren dieses Jahrhunderts - wie zuvor ausgeführt - konnten Peyton Rous u. Mitarb. aufzeigen, daß Teerpinselung der Haut, oder später auch die systemische Anwendung chemischer Kanzerogene, den tumorigenen Effekt von Papillomvirus-Infektionen mit dem WildkaninchenPapillomvirus (cottontail rabbit papillomavirus - CRPV) erheblich steigern konnten. Virus-positive Tumoren erschienen früher, in höherer Frequenz und konvertierten rascher zu malignem Wachstum.

In den Folgejahren wurden weitere Wechselwirkungen zwischen Papillomvirus-Infektionen und chemischen oder physikalischen Kanzerogenen beschrieben, einige davon auch nach Infektionen des Menschen. Ein bei afrikanischen Nagern, Mastomys natalensis, vorkommendes Papillomvirus kann durch tumorpromovierende Chemikalien aktiviert werden. Papillomvirus-positive Zungenpapillome bei Syrischen Hamstern lassen sich durch wiederholte Wundsetzung induzieren. Kehlkopfpapillomatosen des Menschen, die besonders im frühen Kindesalter auftreten und hier lebensbedrohende Atmungsbeschwerden auslösen können, wurden in vergangenen Jahrzehnten gelegentlich mit Röntgenstrahlen behandelt. Diese Papillomatose wird vorwiegend durch die genitalen Papillomvirus-Typen HPV11 und 6 ausgelöst. Viele dieser unglücklichen Kinder entwickelten später Kehlkopfkarzinome, die offensichtlich durch ein Zusammenwirken von Virusinfektion und Röntgenstrahlen ausgelöst wurden.
Heute ist besonders im Brennpunkt die mögliche Wechselwirkung von Papillomvirus-Infektionen der Haut mit dem UVAnteil des Sonnenlichts. Schon bei der Epidermodysplasia verruciformis zeigt sich, daß virushaltige Karzinome ganz überwiegend an lichtexponierten Körperstellen entstehen. Auch unter langwährender Immunsuppression treten Warzen bevorzugt an solchen Stellen auf, ebenso wie Plattenepithelund Basalzellkrebse. Der Nachweis von kutanen Papillomvirus-Typen in den letzteren Tumoren wirft die Frage auf, inwieweit auch hier eine Wechselwirkung mit der Sonneneinstrahlung vorliegt. Interessanterweise enthalten die Promotorregionen vieler dieser HPV-Typen Sequenzen, die vermutlich durch UV-Licht aktiviert werden können.

\section{Präventive Impfung}

Die Identifizierung spezifischer HPV-Typen als Karzinogene für den Menschen wie auch die hohe globale Inzidenz des Gebärmutterhalskrebses (11,8\% aller Krebserkrankungen bei Frauen) und die noch deutlich höhere seiner Vorstufen, führten zu Bemühungen, geeignete Impfstoffe zur Vorbeugung dieser Virusinfektionen zu entwickeln. Gegenwärtig geben diese Bemühungen zu beträchtlichen Hoffnungen Anlaß: Durch die Verwendung gentechnologisch hergestellter viraler Kapsid-Präparationen, wobei es sich entweder um spontan Partikel-bildende L1 oder L1 und L2 Proteine handelt, ließ sich in Tierexperimenten eine bemerkenswerte Wirksamkeit aufzeigen. Hunde wurden komplett gegen das Auftreten von Zungen- und Mundschleimhautpapillomen geschützt, die in Hundezwingern epidemisch auftreten können. Ebenso ließen sich Kaninchen gegen die Infektion mit dem CRPV schützen. In diesem System verhindert die Vaccine auch die Entwicklung maligner Tumoren, besitzt also einen KrebsSchutzeffekt.

Zur Impfung von Menschen werden gegenwärtig auf analoger Basis virusähnliche Partikel für die Typen HPV6, 11, 16 und 18 produziert, wobei sich eine dieser Vakzinen bereits in der klinischen Prüfung befindet. Ihr praktischer Einsatz kann in den kommenden Jahren erwartet werden. Wir stehen daher vor der Anwendung einer spezifischen Krebsschutzimpfung, die neben der Hepatitis-B-Schutzimpfung potentiell in signifikantem Umfang verbreitete menschliche Krebserkrankungen verhüten kann.

\section{Therapeutische Impfstoffe}

Auf der Grundlage ähnlicher Betrachtungen wie im vorausgegangenen Abschnitt werden zur Zeit auch antigene Epitope der viralen Onkoproteine E6 und E7 entwickelt, entweder als Polypeptid-Vakzinen, als chimäre Proteine, wobei sie in die L1-Proteinsequenzen integriert werden, oder als DNA-Vakzinen. Diese Impfung soll den Wirt dann auch im Fall einer bereits vorliegenden Infektion schützen. Die technischen Voraussetzungen hierfür sind inzwischen gut vorangekommen, eine erste klinische Phase-I-Studie wurde bereits publiziert.

Gegenwärtig ist es schwierig, die Wirksamkeit dieser Ansätze abzuschätzen. In den HPV-positiven Karzinomträgern ist trotz intensiver Expression vor allem des E7-Onkoproteins das Immunsystem nicht in der Lage, dieses Protein in den Karzinomzellen als fremd zu erkennen. Dies ist vermutlich ein Problem der fehlenden Präsentation antigener Domänen 
über das MHC-System und erfordert weitere wissenschaftliche Abklärung.

Die Produktion und Anwendung chimärer Proteine mit präventivem und immuntherapeutischem Potential stellen gegenwärtig eine besonders vielversprechende Entwicklung dar. Die theoretischen Einsatzmöglichkeiten solcher Impfstoffe lassen für die Zukunft viel erhoffen.

\section{Chemotherapie}

Die Chemotherapie von Papillomvirusinfektionen befindet sich in einer frühen Phase ihrer Entwicklung. In präklinischen Experimenten sind einige Substanzen in der Lage, die Papillomvirus-Transkription effektiv zu unterdrücken. Hierzu gehören neben der Retinoinsäure (Geisen et al., 1997) auch Antioxidantien, wie das Pyrrolidin-dithio-Carbamat (Rösl et al., 1994), beide wirken vorwiegend in immortalisierten Zellen. Desoxyglucose wirkt dagegen unter GewebekulturBedingungen als Transkriptionssuppressor sowohl in HPVimmortalisierten als auch in malignen Zellen (Maehama et al., im Druck).

Eine Reihe von Zytokinen ließ sich ebenfalls als Transkriptionssuppressoren identifizieren. Hier sind TNF $\alpha$, TGF $\beta$, einige Interleukine (insbesondere Interleukin 6) und Leukoregulin zu nennen. Nach meiner Kenntnis wurde keine dieser Substanzen einer entsprechenden klinischen Prüfung unterzogen. Es könnte sich als interessant erweisen, daß die konstitutive Expression eines Chemokins, des makrophage-chemoattractant protein 1 (MCP-1), in Zervixkarzinom-Zellen deren Tumorigenität im Nacktmaussystem partiell unterdrückt. Es könnte bedeutsam sein, den klinischen Effekt der MCP-1-Verabreichung, möglicherweise unter gentherapeutischer Applikation, zu untersuchen.

Obwohl zahlreiche Versuche unternommen wurden, die klinische Wirksamkeit von Interferon-Präparationen bei Papillomvirus-Infektionen aufzuzeigen, sind diese Versuche insgesamt wenig befriedigend verlaufen.

\section{Schlußbemerkung}

Die Papillomvirus-Forschung hat in den vergangenen 20 Jahren nach jahrzehntelanger Stagnation rasche Fortschritte gemacht. Die Identifizierung krebsverursachender Papillomvirus-Typen beim Menschen und das partielle Verständnis ihrer Wirkungsweise bedingen ihre Akzeptanz als menschliche Kanzerogene. Zweifellos bleibt eine große Zahl von Fragen offen: Wir verstehen nicht die Ursache der enormen Vielfalt von unterschiedlichen HPV-Typen, ihre Rolle bei nicht-genitalen Krebserkrankungen bedarf der weiteren Abklärung, ebenso wie ihre Wechselwirkung mit chemischen oder physikalischen Kanzerogenen. Diese und viele andere Fragen bleiben derzeit unbeantwortet.

Die wohl bemerkenswerteste Konsequenz der PapillomvirusForschung - neben der Krebsursachenforschung - ist die gegenwärtig voranschreitende Entwicklung geeigneter Impfstoffe, die zumindest auf der Basis von Tierexperimenten exzellente Voraussetzungen für einen erfolgreichen Einsatz auch beim Menschen bieten sollten.

\section{Literatur}

Berkhout, R. J. M., L. M. Tieben, H. L. Smits, J. N. Bouwes-Bavinck, B. J. Vermeer, J. Schegget: Detection and typing of epidermodysplasia verruciformis-associated human papillomavirus types in cutaneous cancers from renal transplant recipients: a nested approach. J. Clin. Microbiol. 33 (1995) 690-695.

Bosch, F., E. Schwarz, P. Boukamp, N. E. Fusenig, D. Bartsch, H. zur Hausen: Suppression in vivo of human papillomavirus type 18 E6-E7 gene expression in nontumorigenic HeLa. fibroblast hybrid cells. J. Virol. 64 (1990) $4743-4754$.

Boshart, M., L. Gissmann, H. Ikenberg, A. Kleinheinz, W. Scheurlen, H. zur Hausen: A new type of papillomavirus DNA, its presence in genital cancer and in cell liness derived from genital cancer. EMBO J. 3 (1984) 1151 - 1157.

Ciuffo, G.: Innesto positivo con filtrado di verrucae volgare. G. Ital. Mal. Venerol. 48 (1907) 12.

Crawford, L. V., E. M. Crawford: A comparative study of polyoma and papilloma viruses. Virology 21 (1963) 258-263.

de Villiers, E.-M., D. Lavergne, K. McLaren, E. C. Benton: Preavailing papillomavirus types in non-melanoma skin carcinomas of the skin in renal allograft recipients. Int. J. Cancer 73 (1997) 356 360.

Dürst, M., L. Gissmann, H. Ikenberg, H. zur Hausen: A papillomavirus DNA from a cervical carcinoma and its prevalence in cancer biopsy samples from different geographic regions. Proc. Nat. Acad. Sci. U.S. 80 (1983) $3812-3815$.

Dyson, N., P. M. Howley, K. Münger, E. Harlow: The human papillomavirus-16 E7 oncoprotein is able to bind to the retinoblastoma gene product. Science 243 (1989) 934-937.

Geisen, C., C. Denk, B. Gremm, C. Baust, A. Karger, W. Bollag, E. Schwarz: Highlevel expression of the retinoic acid receptor $\beta$ gene in normal cells of the uterine cervix is regulated by the retinoic acid receptor $\alpha$ and is abnormally down-regulated in cervical carcinoma cells. Cancer Res. 57 (1997) 1460-1467.

Gissmann, L., H. zur Hausen: Human papilloma viruses: physical mapping and genetic heterogeneity. Proc. Nat. Acad. Sci. U.S.A. 73 (1976) $1310-1313$.

Gissmann, L., H. Pfister, H. zur Hausen: Human papilloma viruses (HPV): Characterization of four different isolates. Virology 76 (1977) 569-580.

Gissmann, L., H. zur Hausen: Partial characterization of viral DNA from human genital warts (condylomata acuminata). Int. J. Cancer 25 (1980) 605-609.

Gissmann, L., V. Diehl, H. Schultz-Coulon, H. zur Hausen: Molecular cloning and characterization of human papillomavirus DNA from a laryngeal papilloma. J. Virol. 44 (1982) 393-400.

IARC Monograph on the Evaluation of Carcinogenic Risks to Humans. Vol. 64, Human Papillomaviruses. IARC, Lyon (1995).

Ito, Y.: A tumor-producing factor extracted by phenol from papillomatous tissue (Shope) of cottontail rabbits. Virology 12 (1960) 596-601.

Ito, Y., C. A. Evans: Induction of tumors in domestic rabbits with nucleic acid preparations from partially purified Shope papilloma virus and from extracts of the papillomas of domestic and cotton tail rabbits. J. Exp. Med. 114 (1961) 485-491.

Lewandowsky, T., W. Lutz: Ein Fall einer bisher nicht beschriebenen Hauterkrankung (Epidermodysplasia verruciformis). Arch. Dermatol. Syphil. 141 (1922) 193-198.

Maehama, T., A. Patzelt, H. Lengert, K.-J. Hutter, K. Kanazawa, H. zur Hausen, F. Rösl: Selective down-regulation of the human papillomavirus transcription by 2-deoxyglucose. Int. J. Cancer, in print.

McDougall, J. K.: Immortalization and transformation of human cells by human papillomavirus. In H. zur Hausen (ed.): Current Topics in Microbiology and Immunology. Springer Verlag, Berlin, Heidelberg 86 (1994) $101-119$. 
Orth, G., M. Favre, O. Croissant: Characterization of a new tape of human papillomavirus that causes skin warts. J. Virol. 24 (1977) 108-120.

Orth, G., S. Jablonska, M. Jarzabek-Chorzelska, G. Rzesa, S. Obalek, M. Favre, O. Croissant: Characteristics of the lesions and risk of malignant conversion as related to the type of the human papillomavirus involved in epidermodysplasia verruciformis. Cancer Res. 39 (1979) 1074-1082.

Rösl, F., M. Lengert, J. Albrecht, K. Kleine, R. Zawatzky, B. Schraven, H. zur Hausen: Differential regulation of the JE gene encoding the monocyte chemoattractant protein (MCP-1) in cervical carcinoma cells and derived hybrids. J. Virol. 68 (1994) 2142 2150.

Rous, P., J. W. Beard: Carcinomatous changed in virus-induced papillomas of the skin of rabbits. Prod. Soc. Exp. Biol. \& Med. 32 (1934) $578-580$.

Rous, P., J. W. Beard: The progression to carcinoma of virus induced rabbit papillomas (Shope). J. Exp. Med. 62 (1935) 523-548.

Rous, P., W. F. Friedewald: The effect of chemical carcinogens on virus-induced papillomas (Shope). J. Exp. Med. 79 (1944) 511 538.

Schwarz, E., U. K. Freese, L. Gissmann, W. Mayer, B. Roggenbuck, H. zur Hausen: Structure and transcription of human papillomavirus type 18 and 16 sequences in cervical carcinoma cells. Nature 314 (1985) $111-114$.

Shamanin, V., M. Glover, C. Rausch, C. Proby, I. M. Leigh, H. zur Hausen, E. M. de Villiers: Specific types of HPV found in benign proliferations and in carcinomas of the skin in immunosuppressed patients. Cancer Res. 54 (1994) 4610-4613.

Shamanin, V., H. zur Hausen, D. Lavergne, C. Proby, I. M. Leigh, C. Neumann, H. Hamm, M. Goos, U.-F. Haustein, E. G. Jung, G. Plewig, H. Wolff, E.-M. de Villiers: Human papillomavirus infections in non-melanoma skin cancers from renal transplant recipients and nonimmunosuppressed patients. J. Natl. Cancer. Inst. 88 (1996) 802-811.

Shope, R. E.: Infectious papillomatosis of rabbits. J. Exp. Med. 58 (1933) 607-627.

Strauss, M. J., E. W. Shaw, H. Bunting, J. L. Melnick: "Crystalline" virus-like particles from skin papillomas characterized by intranuclear inclusion bodies. Proc. Soc. Exp. Biol. Med. 72 (1949) 46-50.

Werness, B. A., A. J. Levine, P. M. Howley: Association of human papillomavirus types 16 and 18 E6 proteins with p53. Science 248 (1990) 76-79.

zur Hausen, H., W. Meinhof, W. Scheiber, G. W. Bornkamm: Attempts to detect virus-specific DNA sequences in human tumors: I. Nucleic acid hybridizations with complementary RNA of human wart virus. Int. J. Cancer 13 (1974a) 650-656.

zur Hausen, H.: Condylomata acuminata and human genital cancer. Cancer Res. 36 (1976) 530.

zur Hausen, $\mathrm{H}$.: Cell-virus gene balance hypothesis of carcinogenesis. Behring Inst. Mitt. 61 (1977) 23-30.

zur Hausen, H.: Intracellular surveillance of persisting viral infections: Human genital cancer resulting from failing cellular control of papillomavirus gene expression. Lancet 2 (1986) 489-491.

zur Hausen, H.: Disrupted dichotomous intracellular control of human papillomavirus infection in cancer of the cervix. Lancet 343 (1994) 955-957.

zur Hausen, H.: Papillomavirus infections - a major cause of human cancers. Biochem. Biophys. Acta, Rev. on Cancer 1288 (1996) F55 -F78.

Eine zusammenfassende Literaturzusammenstellung über Papillomviren und Krebs kann in der als letzter Referenz angegebenen Übersichtsarbeit gefunden werden.

\section{H. zur Hausen}

Deutsches Krebsforschungszentrum

Im Neuenheimer Feld 280

69120 Heidelberg 